\title{
New developments in the simulation of Rutherford backscattering spectrometry in channeling mode using arbitrary atom structures
}

\author{
Jin, Xin
}

2020-10

Jin , X, Crocombette , J-P , Djurabekova , F , Zhang , S , Nordlund , K, Garrido , F \& Debelle , A 2020 , ' New developments in the simulation of Rutherford backscattering spectrometry in channeling mode using arbitrary atom structures ' , Modelling and Simulation in Materials Science and Engineering , vol. 28 , no. 7 , 075005 . https://doi.org/10.1088/1361-651X/ab81a9

http://hdl.handle.net/10138/322699

https://doi.org/10.1088/1361-651X/ab81a9

acceptedVersion

Downloaded from Helda, University of Helsinki institutional repository.

This is an electronic reprint of the original article.

This reprint may differ from the original in pagination and typographic detail.

Please cite the original version. 


\title{
New developments in the simulation of Rutherford backscattering spectrometry in channeling mode using arbitrary atom structures
}

\author{
Xin Jin ${ }^{1,2}$, Shuo Zhang ${ }^{3}$, Jean-Paul Crocombette ${ }^{4}$, Kai Nordlund ${ }^{5}$, Flyura Djurabekova ${ }^{5}$, \\ Frédérico Garrido ${ }^{1}$, Aurélien Debelle ${ }^{1}$
}

${ }^{1}$ Laboratoire de Physique des deux infinis Irène Joliot Curie, CNRS/IN2P3, Université Paris-Saclay, 91405 Orsay, France

${ }^{2}$ Institut de Recherche sur les Céramiques (IRCer), CNRS UMR 7315, Université de Limoges, Centre Européen de la Céramique, 12 rue Atlantis, 87068 Limoges, France

${ }^{3}$ School of Nuclear Science and Technology, Lanzhou University, Lanzhou, Gansu Province 730000, People's Republic of China

${ }^{4} C E A, D E N$, Service de Recherche de Métallurgie Physique (SRMP), Université Paris-Saclay, 91191 Gif-sur-Yvette, France

${ }^{5}$ Department of Physics and Helsinki Institute of Physics, University of Helsinki, P.O. Box 43, FIN00014 Helsinki, Finland

\begin{abstract}
As Rutherford Backscattering Spectrometry in Channeling mode (RBS/C) is an efficient technique for characterizing crystallographic defects, its computational simulation has drawn attention over the past several decades. Recently, a RBS/C simulation code based on the Binary Collision Approximation (BCA) called Rutherford Backscattering Simulation in Arbitrary Defective Crystals (RBSADEC) has been suggested and successfully applied to predict the RBS/C spectra from different damaged materials, whose structures were generated in high-dose ion irradiation atomistic simulations. In the present paper, we introduce new developments improving the flexibility of the developed software and its applicability to different types of materials. More precisely, we modified the algorithm describing the slowdown process of backscattered ions, added fitting parameters in the collision partner search routine, modified the routine taking into account target atom thermal vibrations and provided new descriptions of the ion beam divergence. As an example, the effect of the modifications on simulated RBS/C spectra is shown for an $<011>$-oriented $\mathrm{UO}_{2}$ crystal analyzed with a $3.085 \mathrm{MeV} \mathrm{He}^{2+}$ ion beam. Some of these changes proved necessary to achieve satisfying agreement between simulations and experimental data. Similar observation was made for $<001>$-oriented $\mathrm{Si}$ and $<001>$-oriented GaAs crystals analyzed with a $1.4 \mathrm{MeV} \mathrm{He}^{+}$ion beam. In these simulations, the modifications have also resulted in good agreement with experiment.
\end{abstract}




\section{Introduction}

Rutherford Backscattering Spectrometry (RBS) is a quantitative, usually non-destructive, depthresolved and heavy elements sensitive ion beam analysis technique for analyzing solid materials in the near-surface region [1]. In a particular RBS technique configuration that is called RBS in Channeling mode (RBS/C), the incoming ion beam is aligned with one of the major target crystal directions. This technique is widely used to study the crystallographic defects, the knowledge of which is indispensable in many applications related, for instance, to the nuclear energy and semiconductor industries $[2,3]$.

Over the past decades, several Monte-Carlo simulation programs of RBS/C spectra have been developed [4-10]. The basic principle is to follow trajectories of incident ions in the target along the channeling direction or under the random configuration. In this process, random samplings are usually performed on initial positions of the incident ion trajectories, target atom thermal vibrations, incident ion initial directions, etc. Interactions of incident ions with target atoms are treated based on either the Binary Collision Approximation (BCA) alone [4,7-10] or on the combination of BCA and the continuum string approximation [5,6]. In the latter method, the potential formed by individual atoms in an adjacent row or plane is replaced by an averaged potential according to the continuum string approximation [11]. Hence, the influence of the surrounding atoms on the moving atom can be taken into account more accurately compared to the BCA algorithm. However, the necessity of having the analyzed structure in form of continuous rows or planes in the continuum model imposes stronger limitations on the choice of target structures.

Among the above-mentioned simulation programs, a recently developed code Rutherford Backscattering Simulation in Arbitrary Defective Crystals (RBSADEC) has a unique virtue in terms of generating RBS/C signals from crystal targets containing arbitrary atomic structures. These structures can be obtained in molecular dynamics (MD) simulations or by generating atomic structures in some predefined geometry (e.g. stacking fault planes in a crystal). For instance, the code has been used to simulate RBS/C spectra generated from $\mathrm{Ni}$, quartz and two concentrated solid solution alloys, $\mathrm{NiFe}$ and $\mathrm{NiCoCr}$ $[10,12,13]$. For damaged samples, the defects were introduced either by specifying the defect type initially or by simulating recoil-induced cascades in MD cells [10], and then the simulated targets were generated by connecting MD cells corresponding to different irradiation doses to reproduce the damage depth profile [13]. The simulated spectra showed agreement with experimental spectra obtained from both pristine and irradiated samples $[10,12,13]$, which allowed ascribing a defect structure and a defect density to a disorder level, which was previously obtained only phenomenologically.

Although the code was found to give valuable insights on damage evolution in some materials, for efficiency of the calculations, it uses approximations, which are valid in some materials, but may impose undesired limitations in others. In particular, we found it to be very important to improve the code to simulate the RBS/C spectra from materials of high importance for the nuclear energy industry such as $\mathrm{UO}_{2}$. Therefore, we modified the code to obtain accurate simulated RBS/C spectra from pristine samples; this achievement will give a solid base for analysis of damage buildup in this material by using the RBS/C technique.

In the current paper, we focus on the slowdown of the backscattered ions and the search of the collision

partners. We introduced new features, which can be considered as alternative algorithms to those originally implemented in RBSADEC. We also modified the input parameters to include more accurately 
the target atom thermal vibrations and the incident ion beam divergence. An $<011>$-oriented $\mathrm{UO}_{2}$ pristine crystal was used as a reference material to test the effects of the alternative approaches on the RBS/C spectra. In order to show that the code performs equally well in other conditions, two complementary materials, namely $<001>$-oriented $\mathrm{Si}$ and $<001>$-oriented GaAs pristine crystals, were also tested. Results for $\mathrm{UO}_{2}$ and for the complementary materials and conclusions are given in the last two sections.

\section{Experiments and simulation approach}

\subsection{Experiments and simulation input data structures}

The <011>-oriented $\mathrm{UO}_{2}$ pristine crystal was characterized by the RBS/C technique, using the ARAMIS ion accelerator of the SCALP platform [14] in Orsay with a ${ }^{4} \mathrm{He}$ beam of $3.085 \mathrm{MeV}$ energy. The random backscattering spectrum was recorded by rotating the crystal target with a tilt angle of $4^{\circ}$ from the main crystallographic axis. A silicon detector located at $165^{\circ}$ was used to detect backscattered ${ }^{4} \mathrm{He}$ ions. The energy resolution of the detector was $15 \mathrm{keV}$. The $<001>$-oriented $\mathrm{Si}$ and the $<001>$-oriented GaAs crystals were also characterized under the same configuration except that the ${ }^{4} \mathrm{He}$ beam energy was $1.4 \mathrm{MeV}$ and the detector resolution was $20 \mathrm{keV}$.

In RBSADEC simulations, the pristine $\mathrm{UO}_{2}$ target was defined by a box with the $\mathrm{z}$ axis along the $\langle 011\rangle$ direction. The atom coordinates were set according to the perfect crystal. The dimension along the $\mathrm{z}$ axis direction (the depth of the box) was $3 \mu \mathrm{m}$ and periodic boundary conditions were applied in the $\mathrm{x}$ and $\mathrm{y}$ directions. The pristine Si and GaAs targets were defined by the same method. However, we found that the experimental Si and GaAs samples were not perfect, and hence Randomly Displaced Atoms (RDA) were introduced in the simulation to account for defects in the real samples. The RDA fraction along the depth was around $1 \%$ in the Si target and was around $1.7 \%$ in the GaAs target. The simulation depths in the $\mathrm{Si}$ and GaAs targets were $1.2 \mu \mathrm{m}$.

\subsection{Simulation approach}

The detailed description of algorithms implemented in RBSADEC can be found elsewhere [10]. In this paper, we briefly outline the general simulation approach and focus on the new features implemented to improve the code for a broader range of materials.

The interactions between the ions and the target atoms in RBSADEC are calculated using the ZieglerBiersack-Littmark (ZBL) universal interatomic potential [15]. The target is read from a file containing the three-dimensional coordinates of the target atoms. A probing ion searches for collision partners according to a specifically designed search procedure. In the RBSADEC code, the return of the backscattered ions is treated according to the conventional BCA algorithm, in which the actual structure is disregarded and collisions are searched randomly according to the average density of the material. The backscattering event, which may eventually contribute to the RBS/C signal, is triggered according to the encounter probability [4].

The description of the new features is organized as follows. Before presenting the alternative simulation approach, we provide a brief description of the algorithm for the given process and its original implementation in RBSADEC. The features are presented in order according to the importance of their effect on RBS/C spectra. 


\title{
2.2.1 Slowdown of backscattered ions
}

\author{
a. Simulation algorithm
}

The process of searching for a partner for the collision from the read-in coordinates of the actual structure is a time-consuming procedure. In order to simulate statistically meaningful RBS/C signal, the number of simulated ions must be very large. Since the probability of the ion to be captured in a channel is not high in a random direction, the use of a BCA search algorithm (assuming an amorphous-like structure) to simulate the slowdown of the backscattered ions on their return to the surface is well justified. This approach is reasonable for many materials, except when the detector direction is aligned with one of the major target crystal directions. In the BCA search algorithm, the trajectory is mainly affected by how the free flight path (FFP), $L$, between two successive elastic collisions and the impact parameter, $P$, are chosen. Hence, while keeping generally the same approach of how a backscattered ion is treated (amorphous slowdown process), we focus on different ways to define the free flight path and the impact parameter for a collision.

In addition, it is worth mentioning that the algorithm of the multiple scattering was implemented in the process of the slowdown of backscattered ions. The discrepancies of different slowdown approaches (in the next section) can be more pronounce under different multiple scattering conditions. The algorithm is illustrated in Figure 1. The initial direction of the backscattered ion is confined in a region defined by a given solid angle. The symmetry axis of the solid angle points to the detector center. The maximum angle between the backscattered ion initial direction and the detector center direction is a preset, fixed value that can be called the spread angle, $\theta_{s}$. The multiple scattering effect can be adjusted by changing $\theta_{s}[16]$.

\section{b. Original approach}

In the original version of RBSADEC, FFP is chosen by a random sampling following a Poisson process with the average value equal to $10 r_{v}$, where $r_{v}$ is the radius of the average atomic volume (on the order of magnitude of an Ångstrom). The probability to have a certain impact parameter $f(P)$ in the collision event linearly increases with $P$ as expressed in the following Probability Density Function (PDF):

$$
f(P)=\frac{2 \pi P L}{r_{v}{ }^{3}} \quad \text { for } 0 \leq P \leq \sqrt{r_{v}{ }^{3} / \pi L}
$$

where the maximum impact parameter, $P_{\max }$, is $\sqrt{r_{v}{ }^{3} / \pi L}$ so that the value of its Cumulative Distribution Function (CDF) is no larger than 1. The maximum impact parameter implies that one target atom can definitely be found in a fixed volume equal to $r_{v}{ }^{3}$. This impact parameter calculation method will be, in the following, denoted as the original fixed-volume method.

\section{c. New approaches}

In the new version of RBSADEC, we applied four alternative approaches. The first one is mainly based on the TRansport of Ions in Matter (TRIM) program[15,17] (denoted as the TRIM-based approach). In TRIM, the FFP is energy dependent and is determined such that the mean angular deflection is about $5^{\circ}$ per path length (see Equation (24) in Ref [17]). It generally increases with energy as shown in Figure 2 with $\mathrm{He}$ ions transported in $\mathrm{UO}_{2}, \mathrm{Si}$ and $\mathrm{GaAs}$ as examples. It can also be found from Figure 2 that the FFP for $\mathrm{MeV} \mathrm{He}$ ions (representative of typical backscattering events) is very large, i.e. on the order of 
one micrometer, so that the initial and final energies of the free flight ion can be very different. The corresponding electronic stopping power from the initial to the final positon of the FFP could, accordingly, vary significantly. Therefore, the electronic energy loss, according to the conventional method, which is equal to the path length times the initial electronic stopping power (unit: energy/length), would be no more appropriate.

In the implementation of the FFP method, we divided each FFP into several sub-FFPs which are no larger than $100 \AA$. In each sub-FFP, the electronic stopping power can be considered as constant, and the electronic energy loss is calculated according to the conventional method. By this way, the electronic stopping power in the next sub-FFP can be updated according to the final energy in the previous sub-FFP, and eventually the electronic energy loss in the total FFP can be calculated properly.

The PDF of the TRIM impact parameter reads:

$$
f(P)=e^{-N L \pi P^{2}} N L 2 \pi P
$$

where $\mathrm{N}$ is the atomic density of the target. In Equation (2), the exponential term represents the probability for not finding another atom closer than $P$, and the term on the right hand side has essentially the same meaning as that of Equation (1) which represents the probability of finding an atom at $P$. This impact parameter calculation method will be denoted as TRIM-exponential method.

In the second Alternative SlowDown approach (denoted as ASD1), we used the original random sampling for the FFP calculation and calculated the impact parameter according to the TRIM-exponential method. For the third one (denoted as ASD2), we also used the TRIM-exponential method to obtain the impact parameter, but the FFP was set as a fixed value (100 $\AA$ ).

The last approach (denoted as ASD3) we applied is based on one of the simulation choices in a TRIMlike BCA simulation code called Iradina $[18,19]$. In this approach, the FFP is fixed as $100 \AA$, and the impact parameter PDF is calculated with the following equation:

$$
f(P)=2 \pi N L P \quad \text { for } 0 \leq P \leq \sqrt{1 / \pi N L}
$$

where the atomic density $\mathrm{N}$ is equal to $1 /\left(4 / 3 \pi r_{v}{ }^{3}\right)$. Equation (3) indicates that one target atom can definitely be found in a fixed volume equal to $4 / 3 \pi r_{v}{ }^{3}$. Since Equation (3) has the same physical form as Equation (1) except for the fixed volume, this impact parameter calculation method will be denoted as the modified fixed-volume method.

Figure 3 shows the PDFs according to the three impact parameter calculation methods in $\mathrm{UO}_{2}$ with the FFP set to $100 \AA$. It can be observed that the TRIM-exponential and modified fixed-volume methods give close results when the impact parameter is small. In contrast, the probabilities according to the original and modified fixed-volume methods are always different. The characteristics of the different amorphous slowdown approaches are summarized in Table 1. 


\title{
2.2.2 Maximum impact parameter in the search of collision partners before backscattering
}

\author{
a. Simulation algorithm
}

In the transportation history of the probing ion, which is different from that of the backscattered ion, target atoms located in front of the probing ion are potential candidates for an elastic collision. This statement can be rephrased as follows: the potential candidates are target atoms with their projection length along the projectile moving direction, $\xi$, larger than 0, as shown in Figure 4. Finally, atoms with the minimum projection length, $\xi_{\min }$, and with an impact parameter, $P$, smaller than the maximum impact parameter, $P_{\max }^{S}$, are chosen as collision partners. (Note the difference with the $P_{\max }$ used in the backscattered ion slowdown process). The cut-off on the $P_{\max }^{S}$ implies that the influence of atoms with larger impact parameter on the trajectory of the projectile can be neglected. In addition, the projectile is not allowed to collide with the last collided target atoms.

\section{b. Original approach}

The original collision partner search process is fitting parameter free, since $P_{\max }^{S}$ is automatically computed according to the target nature. It is in fact the radius of the average target atomic volume.

\section{c. New approach}

Instead of directly adjusting the $P_{\max }^{S}$ cut-off with a distance, we applied an alternative method based on the projectile/target atom interatomic potential energy. By this way, users need to choose the minimum interatomic potential energy, $E_{\min }^{p}$, at which the interaction strength is significant, and then the modified code can compute the corresponding $P_{\max }^{s}$. The derived $P_{\max }^{s}$ is no longer uniform for all elements, and it can hence take into account the different nature of target atoms. The interatomic potential used is also the ZBL universal potential $[15,20]$, and the distance corresponding to the minimum potential energy is solved by Newton's method [21].

\subsubsection{Simultaneous collisions in the search of collision partners before backscattering}

a. Simulation algorithm

$\mathrm{BCA}$ is based on the approximation that the trajectory of the ion can be decomposed in a series of two body collisions. This approximation imposes some limitations, since multibody interactions cannot be taken into account. One way to circumvent partly this limitation is to introduce the so-called simultaneous collisions enhancement to BCA.

To understand the interest of this enhancement, one can consider the projection lengths depicted in Figure 4: the target atom $T_{l}$ has the minimum projection length. It will therefore be selected as a collision partner, but it is possible that a target atom with a larger projection length, for example $T_{2}$, is closer to the projectile. Therefore, in reality, the trajectory of the probing ion is affected (by $T_{2}$ ) before or concurrently with the collision with $T_{1}$. The method called 'simultaneous collisions' tries to mitigate such situations by considering the influence of other atoms with a larger projection distance. It was proposed in MARLOWE [22] and is applied in the original RBSADEC code. In this method, if the difference, $\Delta \xi$, between the projection length of one atom and the minimum projection distance is smaller than the value defined by a 
user, then the atom is considered as one of the collision partner candidates. In the following, this user defined value related to the difference in projection length will be referred to as the projection interval $\Delta \xi_{\max }$. In simultaneous collisions, each collision is treated independently and the final deflection vectors are added together.

b. Original approach

The value of $\Delta \xi_{\max }$ is always set to zero. The simultaneous collisions enhancement is therefore not activated.

c. New approach

Considering that the effect of the simultaneous collisions enhancement on RBS/C spectra has been seldom discussed, we introduced one fitting-parameter for this particular process by enabling the adjustment of $\Delta \xi_{\text {max }}$ to enhance the fit quality.

\subsubsection{Target atom thermal vibrations}

a. Simulation algorithm

Target atom thermal vibrations are described by uncorrelated Gaussian distributions. The vibration magnitudes are important initial parameters for determining the projectile trajectory and the final spectrum of backscattered particles.

\section{b. Original approach}

As applied in some other channeling programs [4,23,24], the one-dimensional (1D) root mean square (rms) vibration magnitude is calculated based on the Debye theory. Users need to input the target Debye temperature and the environment temperature. In this code, the vibration magnitude value is solved by an analytical method, which is close to the numerical solution when the ratio of Debye temperature over the environment temperature is not too large [25].

c. New approach

Considering that the $1 \mathrm{D}$ rms vibration magnitude can alternatively be derived from other methods including the "B factor" or the "temperature factor" related to the Debye-Waller parameter [26] and channeling experiments [27,28], we let users be able to input the vibration magnitude directly. Note that with this approach, the magnitude can be defined independently for each element of a compound.

\subsubsection{Incident ion beam divergence}

a. Simulation algorithm

In RBSADEC, the incident ion is emitted from a parallel plane in front of the target surface. The initial position is uniformly distributed over the incident plane. The incident ion beam divergence (IBD) is used 
to account for the angular spread of the probing beam and for the target surface disorder. It is also an important initial parameter for the final backscattering spectrum.

b. Original approach

The ion beam divergence is considered to have a uniform distribution inside a given solid angle region. If the ion beam angular profile is described in spherical coordinates and the minimum and maximum polar angles are set to 0 and $\theta_{\max }$, then the PDF of the polar angle $\theta$ can be expressed by the following equation:

$$
P(\theta)=\frac{1}{\left(1-\cos \theta_{\max }\right)} \sin \theta \text { for } 0 \leq \theta \leq \theta_{\max }
$$

The original divergence approach will be denoted as "IBD type A".

c. New approaches

We added two other angular profile methods into the code. The angular profile of the first one (IBD type B) exhibits a Gaussian distribution along the polar angle:

$$
P(\theta)=\frac{2}{\sqrt{2 \pi \sigma_{1}^{2}}} e^{-\frac{\theta^{2}}{2 \sigma_{1}^{2}}} \text { for } \theta \geq 0
$$

where $\sigma_{1}$ is the standard deviation of the Gaussian distribution. The second one (IBD type C) is expressed as:

$$
P(\theta)=\frac{1}{\sigma_{2}^{2}} e^{-\frac{\theta^{2}}{2 \sigma_{2}^{2}}} \theta \quad \text { for } \theta \geq 0
$$

where $\sigma_{2}$ is the characterization angle for the profile. This special angular profile can lead to the PDF of the ion spatial profile exhibiting a Gaussian distribution along the $\mathrm{x}$ and $\mathrm{y}$ directions on the target surface (more details can be found in the Appendix). In addition, for all the divergence methods, the distribution along the azimuthal angle is uniform.

\section{Results and Discussion}

\subsection{Slowdown process of backscattered ions}

The effect of how a free flight path and an impact parameter for a collision are defined for a backscattered ion during its return (amorphous slowdown process) will be presented by comparing RBS/C random spectra. The simulated random spectra were obtained by rotating the incident ion beam center with a tilt angle of $4^{\circ}$ from the normal to the target surface, while the ion beam center azimuthal angle is repeated from $0^{\circ}$ to $359^{\circ}$ with $1^{\circ}$ increase for each ion.

a. Comparison of the original and TRIM-based approaches

Figure 5 shows simulated RBS/C spectra of $3.085 \mathrm{MeV} \mathrm{He}$ on $\mathrm{UO}_{2}$ in a random geometry using different spread angles applied to the initial direction of the backscattered ions. An experimental spectrum is also plotted in this figure. The simulated spectra in the upper part are calculated based on the RBSADEC 
original slowdown approach, and the simulated spectra in the bottom part are based on the TRIM slowdown approach.

Spectra based on both approaches exhibit a similar trend that is an increase in the normalized yield in the low energy region with an increase in the spread angle. This findings can be explained with the help of Figure 1. When the spread angle is increased, more of the collected backscattered ions would have their initial direction away from the detector direction. If these ions still reach the detector, they would experience overall deflections on average larger than those whose initial direction was closer to the direction of the detector and thus the detected energy of the former would be lower.

A distinct difference between the two approaches does exist, however. For the original one, the RBS/C spectra with a spread angle higher than $30^{\circ}$ can exceed the experimental spectrum in some energy regions, particularly at low energy, whereas for the TRIM-based approach, the spectra always approach the experimental one, without ever exceeding it. Therefore, the spectra from the TRIM-based approach provide a better fit to the experimental spectrum.

b. Comparison of the TRIM-based, ASD1 and ASD2 approaches (for comparing FFP methods)

As shown in Figure 6, by comparing RBS/C spectra from the TRIM-based, ASD1 and ASD2 approaches, it is observed that all simulated spectra show a good agreement with the experimental data, and the three different FFP methods do not affect significantly the output. However, the spectrum from the TRIM method shows an unphysical peak at around $1.3 \mathrm{MeV}$. This is because the FFP, for a $5^{\circ}$ mean angular deflection, is too long, and the moving direction variation is not taken into account until the next collision event. In contrast, the FFPs calculated according to the random sampling and to the fixed value $(100 \AA)$ are short enough to consider that the moving direction variation during the return of the backscattered ion is not important for the final spectra.

c. Comparison of the original and ASD (1-3) approaches (for comparing impact parameter methods)

After we established that the randomly sampled and the fixed values of FFP are equally applicable, we study the effect of choice of the calculation method of the impact parameter by comparing the results obtained by using these two FFP methods. In Figure 6, by comparing the spectra from the original and ASD1 approaches, it can be found that the TRIM-exponential method estimates better the impact parameter compared with the original fixed-volume method. By comparing the spectra from ASD2 and ASD3 approaches, we see that the effect of the TRIM-exponential method is almost the same with that of the modified fixed-volume method.

\section{d. Discussion and summary}

We see that the calculation methods of the FFP and of the impact parameters can significantly affect the simulated RBS/C spectra, especially for low energy backscattered ions that experience multiple scatterings on their way back to the surface. The strongest effect we observed is due to the method used to calculate the impact parameters.

Out of the two fixed volume methods for the calculation of the impact parameters, the modified fixed-

volume method results in a better agreement with experiment. The TRIM-exponential method provides 
equivalent results as those obtained with the modified fixed-volume one. It can be explained by the fact that the TRIM-exponential and modified fixed-volume methods share close agreement when the impact parameter is small, as shown in Figure 3. The effect of the discrepancy in the large impact parameter region can be neglected, since small impact parameter collisions are more important than large impact parameter collisions in terms of the nuclear energy loss and the trajectory deflection.

We also see that the FFP calculation according to the TRIM approach results in a too large value, which gives rise to small unphysical peaks on the spectrum. However, if these unphysical peaks can be ignored, this method allows for faster simulations due to the larger FFP.

Based on our results, we conclude that the perfect agreement of the simulated RBS/C spectra with experiment can be achieved if the chosen FFP calculation method is either the random sampling or the fixed-value method. However, in the latter, too long FFP values should be avoided. We also recommend to use the TRIM-exponential or modified fixed-volume methods to calculate the impact parameter. We note here that the TRIM FFP method can be applied, if the effect of unphysical peaks is small, to increase the computational efficiency of the simulations.

In the following, we will focus on the practical applications of the newly proposed approaches to simulate the RBS/C spectra from $\mathrm{UO}_{2}$ and from two additional materials.

\subsection{Maximum impact parameter and simultaneous collisions in the search of collision partners}

a. Maximum impact parameter

Figure 7 shows the RBS/C aligned spectra from the $3.085 \mathrm{MeV}$ He ion beam along the $\langle 011\rangle$ direction of a pristine $\mathrm{UO}_{2}$. The RBSADEC simulations are performed with different maximum impact parameters. The simulated spectrum denoted $R_{a}$ represents the results obtained with the original approach (fitting parameter free), in which the maximum impact parameter $P_{\max }^{S}$ is equal to the radius of the average atomic volume $\left(P_{\max }^{S}=1.482 \AA\right)$. The other simulated spectra are labeled by the given value of energy that corresponds to the minimum projectile/target atom interatomic potential energy $E_{\min }^{p}$. The $P_{\text {max }}^{s}$ values used in the simulation are the distances from $\mathrm{He}$ to either $\mathrm{U}$ or $\mathrm{O}$ atom, where the interatomic potential energy is equal to $E_{\min }^{p}$. The $P_{\max }^{s}$ values associated to the different $E_{\min }^{p}$ values are given in Table 2.

As shown in Figure 7, the simulated yield of backscattered ions is strongly underestimated while using the original approach as compared with experiment. Decreasing $E_{\min }^{p}$ (equivalent to increasing $P_{\max }^{s}$ ) improves the simulated yield significantly with the best result observed with an $E_{\min }^{p}$ value equal to 0.3 $\mathrm{eV}$.

b. Simultaneous collisions

Figure 8 shows RBS/C aligned spectra, in which the $E_{\min }^{p}$ value is set to $0.01 \mathrm{eV}$. As one can see the original simultaneous collisions approach with no projection interval $(0 \AA)$ overestimates the yield of backscattered ions with respect to the experiments. A satisfactory fit is achieved by setting the projection interval to $3 \mathrm{pm}$.

c. Discussion and summary 
The increase of the simulated yield with a lower $E_{\min }^{p}$ (equivalent to a higher $P_{\max }^{S}$ ) can be explained as follows. Including the weaker interatomic interactions increases the number of atoms that can be taken into account as collision partners, and thus the projectile undergoes more collision events; this increase has a non-negligible contribution to the ion de-channeling. Theoretically, if the influence from more surrounding atoms is taken into account, the simulated spectrum should have a better agreement with the experimental one. However, according to Figure 7, when more target atoms are involved in the simulation with a $E_{\min }^{p}$ value lower than $0.3 \mathrm{eV}$, the simulated spectra agree less (actually overestimate) with the experimental spectrum.

The overestimation problem can be approached by adjusting the fitting parameter in the simultaneous collisions process as shown in Figure 8. However, there is still the problem of over-adjustment if the projection interval is too large. Presumably, the simultaneous collisions algorithm avoids the instability of well-channeled projectiles [22], but on the contrary, it can obstruct the normal de-channeling process in some conditions. Additionally, if there are many target atoms involved in one simultaneous collisions event, one drawback is that the current algorithm of forbidding the collision with the last collided atom becomes questionable. In order to take this problem into account, new user-defined quantity may need to be introduced [29], which magnifies the simulation complexity.

In a short summary, if the simulated aligned spectrum according to the original approach has a nonnegligible discrepancy with the experimental one, we recommend only to adjust the maximum impact parameter by changing the minimum interatomic potential energy, and to keep the original setting for the simultaneous collisions. The use of the simultaneous collisions enhancement is optional but not necessary, since taking into account both the maximum impact parameter and the simultaneous collisions brings more complexity. In the following, we only adjusted the minimum interatomic potential energy, when necessary.

\subsection{Target atom thermal vibrations}

For the atom thermal vibration magnitudes in $\mathrm{UO}_{2}$, the Debye temperature at an environment temperature of $285 \mathrm{~K}$ is calculated to be $614 \mathrm{~K}$ [30]. According to the original approach, the $1 \mathrm{D} \mathrm{rms}$ vibration magnitudes for the $\mathrm{U}$ and $\mathrm{O}$ atoms are found to be $2.2 \mathrm{pm}$ and $8.5 \mathrm{pm}$, respectively. According to the "B factor" or "temperature factor" obtained from neutron diffraction experiments at the same environment temperature [31], the vibration magnitudes for the $\mathrm{U}$ and $\mathrm{O}$ atoms in $\mathrm{UO}_{2}$ are $6.9 \mathrm{pm}$ and 8.5 pm, respectively. In addition, the vibration magnitudes obtained from RBS/C experiments, which are 6.5 $\mathrm{pm}$ for the $\mathrm{U}$ atom and $9 \mathrm{pm}$ for the $\mathrm{O}$ atom [27], are similar to those derived from neutron diffraction experiments. It can be observed from Figure 9 that the spectra based on the magnitudes obtained from the two experiments are similar and fit well the experimental one, but the spectrum based on the Debye theory shows significant discrepancy with the experimental data mainly due to the underestimated $U$ atom thermal vibration magnitude.

The discrepancy between the thermal vibration magnitudes can be ascribed to the fact that the Debye theory works better with monoatomic crystals [26,32]. Therefore, we recommend a user to use the thermal vibration magnitude measured from experiments for each species separately instead of relying totally on the Debye theory only. In all the simulations using a $\mathrm{UO}_{2}$ target in this paper, the $1 \mathrm{D}$ rms vibration 
magnitudes were those derived from the RBS/C experimental results $(6.5 \mathrm{pm}$ for the $\mathrm{U}$ atom and $9 \mathrm{pm}$ for the $\mathrm{O}$ atom).

\subsection{Incident ion beam divergence}

Figure 10 shows the comparison between the experimental spectrum and RBSADEC simulation spectra with different ion beam divergences. Although the three IBD types have different forms in terms of the angular profile, spectra from these three types can still prove to be satisfactory fits to the experimental spectrum by adjusting the characteristic value $\left(\theta_{\max }\right.$ as $0.18^{\circ}$ for the type $\mathrm{A}, \sigma_{1}$ as $0.12^{\circ}$ for the type $\mathrm{B}$ and $\sigma_{2}$ as $0.09^{\circ}$ for the type $C$ ). However, when all the characteristic values are set to the same value $\left(0.12^{\circ}\right)$, the difference among the spectra is not negligible. Hence we suggest that, when spectra obtained by using RBSADEC code are compared with the spectra generated by other codes, the IBD types should be the same, or the characteristic values must be adapted to generate comparable spectra.

\subsection{Tests with Si and with GaAs}

For these materials, the effect of the different methods to define the thermal vibration magnitudes will not be addressed, because in these two complementary materials, on the one hand, $\mathrm{Si}$ is a monoatomic crystal, and on the other hand, for GaAs, the magnitude difference between the results derived from B factors and from the Debye theory is small [33,34]. The 1D rms value for the Si atom is thus set to $8 \mathrm{pm}$ [28], and the values for the $\mathrm{Ga}$ and As atoms are set to $9 \mathrm{pm}$ and $8 \mathrm{pm}$, respectively [33]. In contrast, the effects of the different approaches applied in the amorphous slowdown process and in the collision partners search process (maximum impact parameter) will be tested by comparing RBS/C spectra.

Figure 11(a) shows RBS/C random spectra of $1.4 \mathrm{MeV}$ He on Si from both the experiment and the RBSADEC simulations. In the simulations, two approaches to simulate the amorphous slowdown process (the original approach and the TRIM-based approach) were used. It can be found that the two simulated spectra fit well with the experimental data, thus the improvements suggested in the current paper do not affect the random spectra from Si crystal.

Figure 11(b) shows the RBS/C aligned spectra of $1.4 \mathrm{MeV}$ He on Si along the $<001>$ direction obtained experimentally and in the RBSADEC simulations. In the simulation using the original slowdown approach, we applied the original approach for finding the maximum impact parameter $P_{\max }^{S}$ in the collision partner search process. The $P_{\max }^{S}$ is set to $R_{a}(1.685 \AA)$ by default, which is equivalent to $1.6 \mathrm{eV}$ in terms of the interatomic potential energy. For the simulations using the TRIM-based slowdown approach, the $P_{\max }^{S}$

values are adjusted by the $E_{\min }^{p}$ values $(0.1 \mathrm{eV}$ and $0.6 \mathrm{eV})$. All simulated aligned spectra exhibit a good agreement with the experimental aligned spectra except in the surface peak region. This indicates that, in this case, the sensitivity of aligned spectra on $P_{\max }^{S}$ is not as high as that in the case of $\mathrm{UO}_{2}$ tested above. It is reasonable to use RBSADEC as a fitting-parameter free code in the collision partners search process, as already applied in the original version.

Figure 12(a) shows RBS/C random spectra of $1.4 \mathrm{MeV}$ He on GaAs from both the experiment and the RBSADEC simulations. Figure 12(b) shows RBS/C aligned spectra of $1.4 \mathrm{MeV} \mathrm{He}$ on GaAs along the $<001>$ direction from both the experiment and the RBSADEC simulations. In the simulations, the approaches used are the same with those in Figure 11 for Si target, except that the $P_{\text {max }}^{S}$ set to $R_{a}(1.751$ 
$\AA$ ) is equivalent to $2.0 \mathrm{eV}$ for the $\mathrm{Ga}$ atom and is equivalent to $2.1 \mathrm{eV}$ for the As atom in terms of the interatomic potential energy. Similar conclusions as those derived in the case of Si can be drawn for GaAs.

\section{Conclusions}

In this paper, we improved the applicability and the flexibility of the RBSADEC code for simulating RBS/C spectra under different conditions. New developments were introduced on four aspects: (a) the slowdown process of backscattered ions; (b) the search of collision partners for the probing ion; (c) the target atom thermal vibrations; (d) the incident ion beam divergence. The first three new developments are important for achieving satisfactory fits in as many conditions as possible. First, we recommend that the calculation methods for the free flight path and for the impact parameter should be carefully chosen. The newly proposed impact parameter calculation methods are preferred compared with the original one. Second, we suggest using the new fitting parameter related to the maximum impact parameter (the minimum projectile/target atom interatomic potential energy) to improve the simulated aligned spectrum when necessary. Third, when the studied material is a compound with a large difference in the atomic number of the constituents, we suggest using the atom thermal vibration magnitudes obtained from experiments instead of those derived from the Debye theory. The last new development increases the flexibility of the code by providing new descriptions of the ion beam divergence, which is a useful feature for a relevant comparison with other codes.

The new developments of the code proved particularly important for predicting RBS/C spectra from the materials consisted of atoms with large difference in atomic masses, such as $\mathrm{UO}_{2}$, while for materials with lesser difference in atomic masses, such as pure $\mathrm{Si}$ or GaAs, the original RBSADEC code provides sufficiently good result.

\section{Acknowledgments}

We warmly thank Lionel Thomé for providing the Si and GaAs experimental data. X. Jin is grateful to PHENIICS Doctoral School (Université Paris-Sud/Université Paris-Saclay), Alexandre Boulle and SIMME Doctoral School (Université de Limoges) for the financial support during his PhD study. Part of this work has been funded by the NEEDS program. K. Nordlund acknowledges that his work has also been partly carried out within the framework of the EUROfusion Consortium and has received funding from the Euratom research and training programme 2014-2018 under grant agreement No 633053. The views and opinions expressed herein do not necessarily reflect those of the European Commission.

\section{Appendix. Conversion of the ion beam angular profile to spatial profile}

Considering the incident ion moving direction in spherical coordinates, for the IBD type C, the PDF of the polar angle is expressed in Equation (6):

$$
P(\theta)=\frac{1}{\sigma_{2}^{2}} e^{-\frac{\theta^{2}}{2 \sigma_{2}^{2}}} \theta \quad \text { for } \theta \geq 0
$$

where $\sigma_{2}$ is the characterization angle for the angular profile. As shown in Figure 13, for the sake of simplicity, the initial position of the incident ion is a point source, and the target surface is described in polar coordinates in which the incident ion having normal direction with the target passes the coordinate 
origin. The distance from the point source to the target surface is $\mathrm{d}$, and the radius of the incident ion location on the target surface is $r_{n}$.

When the characterization angle $\sigma_{2}$ is very small, the $r_{n}$ is approximately equal to $\theta \mathrm{d}$. Thus the PDF of $r_{n}$ is expressed as:

$$
P\left(r_{n}\right)=\frac{1}{d^{2} \sigma_{2}^{2}} e^{-\frac{r_{n}^{2}}{2 d^{2} \sigma_{2}^{2}}} r_{n}
$$

After converting the polar coordinates to Cartesian coordinates on the target surface, the PDF of the 2D ion location can now be expressed as:

$$
P(x, y)=\frac{1}{2 \pi d^{2} \sigma_{2}{ }^{2}} e^{-\frac{x^{2}+y^{2}}{2 d^{2} \sigma_{2}{ }^{2}}}
$$

which is a $2 \mathrm{D}$ Gaussian distribution equation. Following the same procedure, the spatial profile of IBD type A and type B on the target surface are shown in following equations respectively:

$$
\begin{gathered}
P(x, y)=\frac{1}{2 \pi\left(1-\cos \theta_{\max }\right) d^{2}} \text { for }|x|<d \theta_{\max } \text { and }|y|<d \theta_{\max } \\
P(x, y)=\frac{1}{\pi \sqrt{2 \pi \sigma_{1}^{2}}} e^{-\frac{x^{2}+y^{2}}{2 d^{2} \sigma_{1}^{2}}} \frac{1}{d \sqrt{x^{2}+y^{2}}}
\end{gathered}
$$

which means that, for IBD type A, the spatial distribution is uniform along the $\mathrm{x}$ and $\mathrm{y}$ direction, and for IBD type $\mathrm{B}$, the probability decreases more rapidly with a higher $\mathrm{x}$ or $\mathrm{y}$ value compared with the Gaussian distribution in the type $C$. In these three ion beam divergence methods, the type $C$ may have a unique advantage, which is that the derived spatial profile on the target surface shows better agreement with those measured in the ion beam characterization experiment [35]. 


\section{Figures}

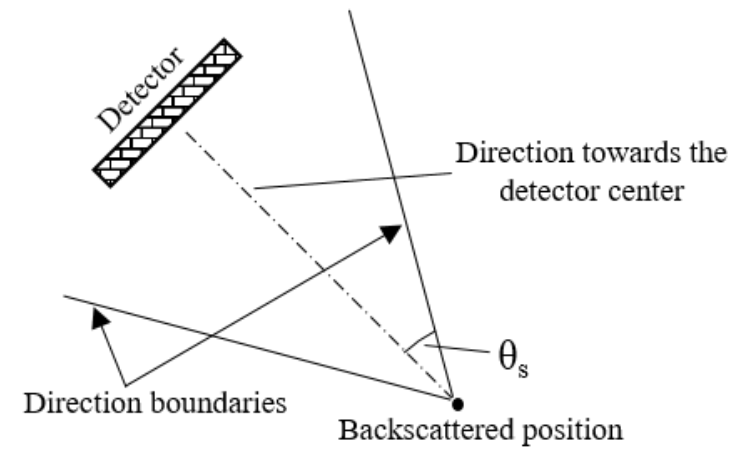

Figure 1. Illustration of the confinement of backscattered ion initial directions.

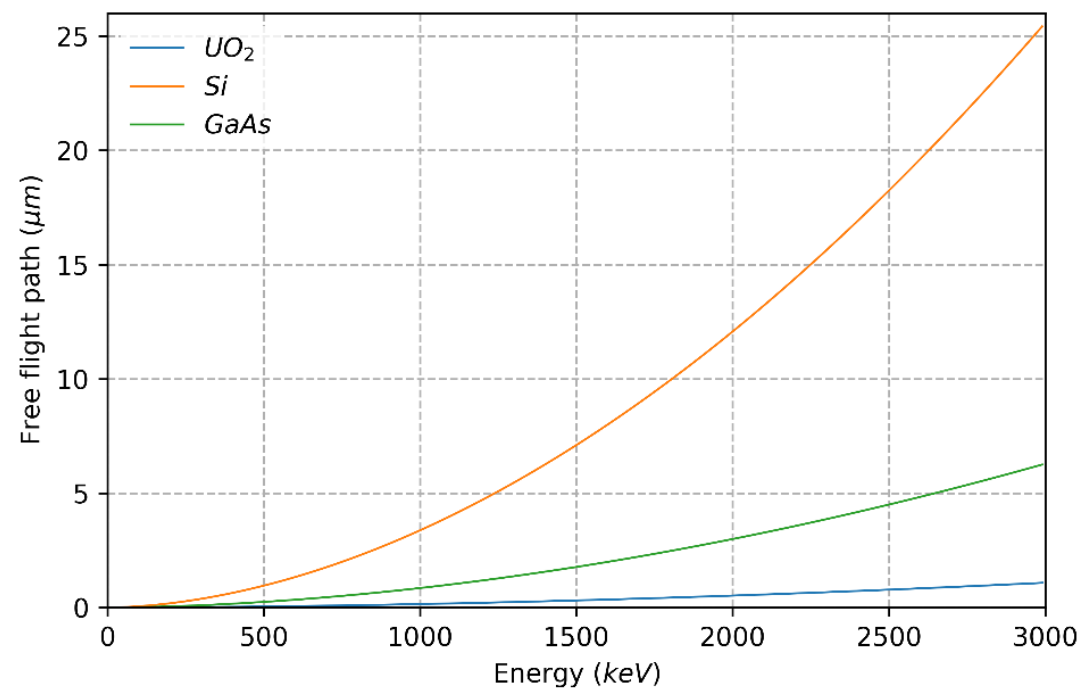

Figure 2. Free flight paths of the $\mathrm{He}$ ion in $\mathrm{UO}_{2}$ (blue), in $\mathrm{Si}$ (orange) and in GaAs (green) calculated according to the TRIM approach. 


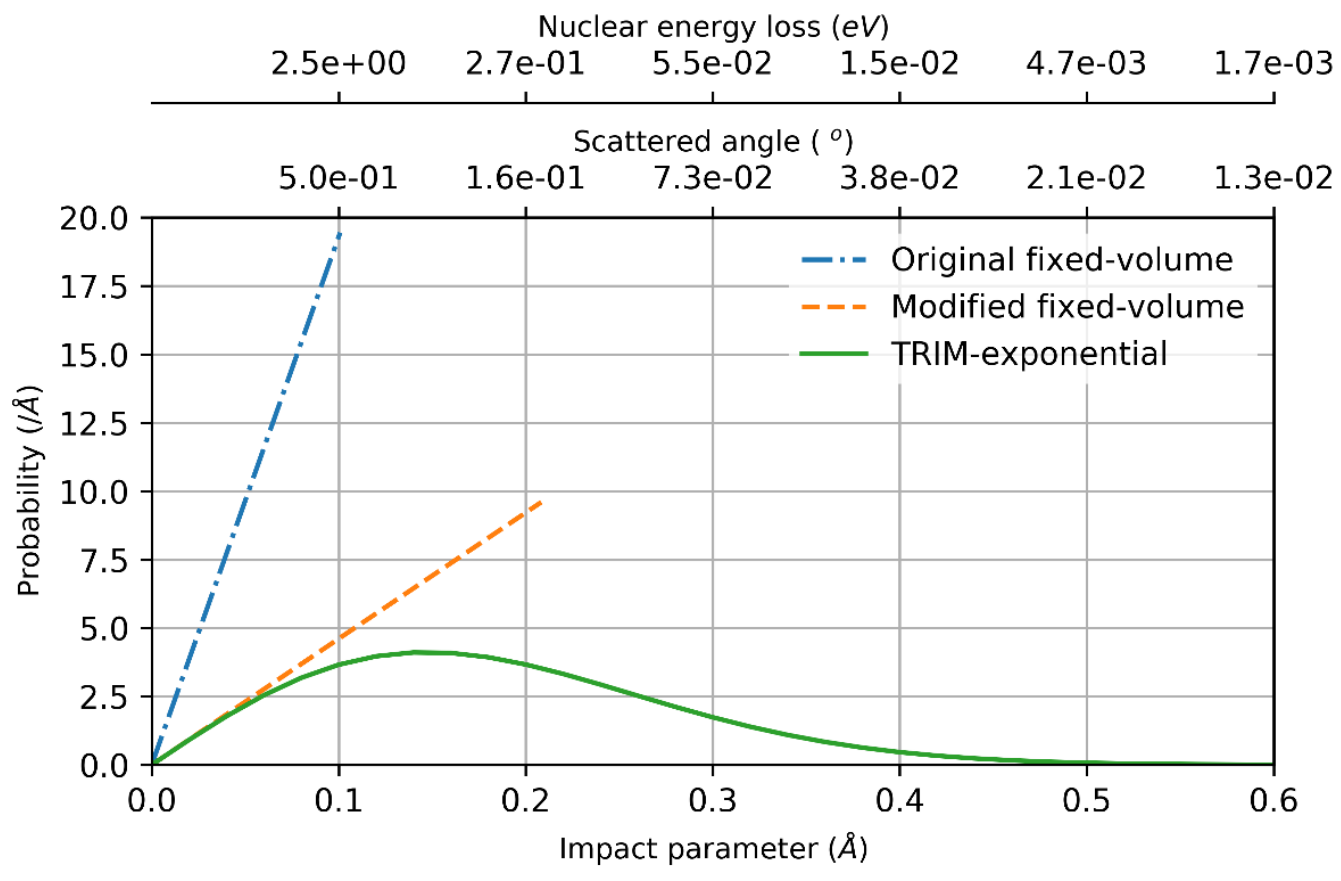

Figure 3. The Probability Density Functions in $\mathrm{UO}_{2}$ with the FFP equal to $100 \AA$. (The upper two axes represent the scattered angle and nuclear energy loss in the elastic collision event of $2 \mathrm{MeV} H e$ ions with U atoms.)

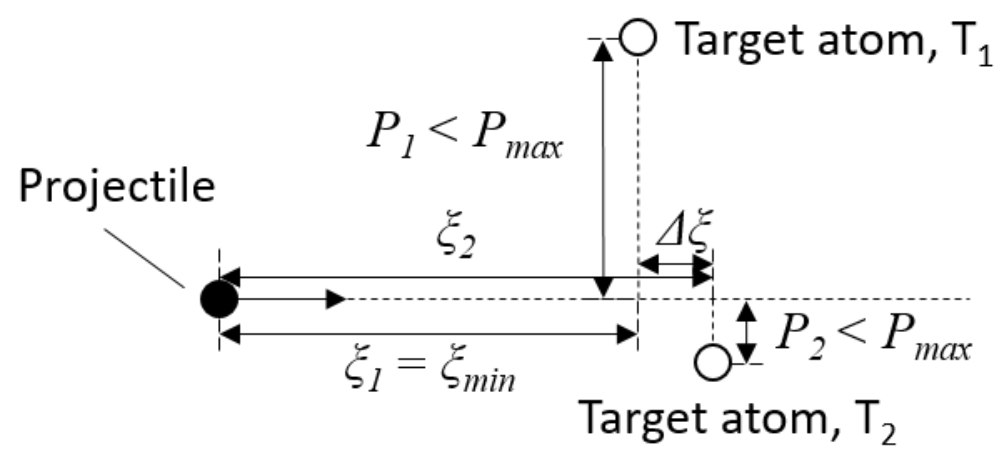

Figure 4. Illustration of the collision partners searching process 


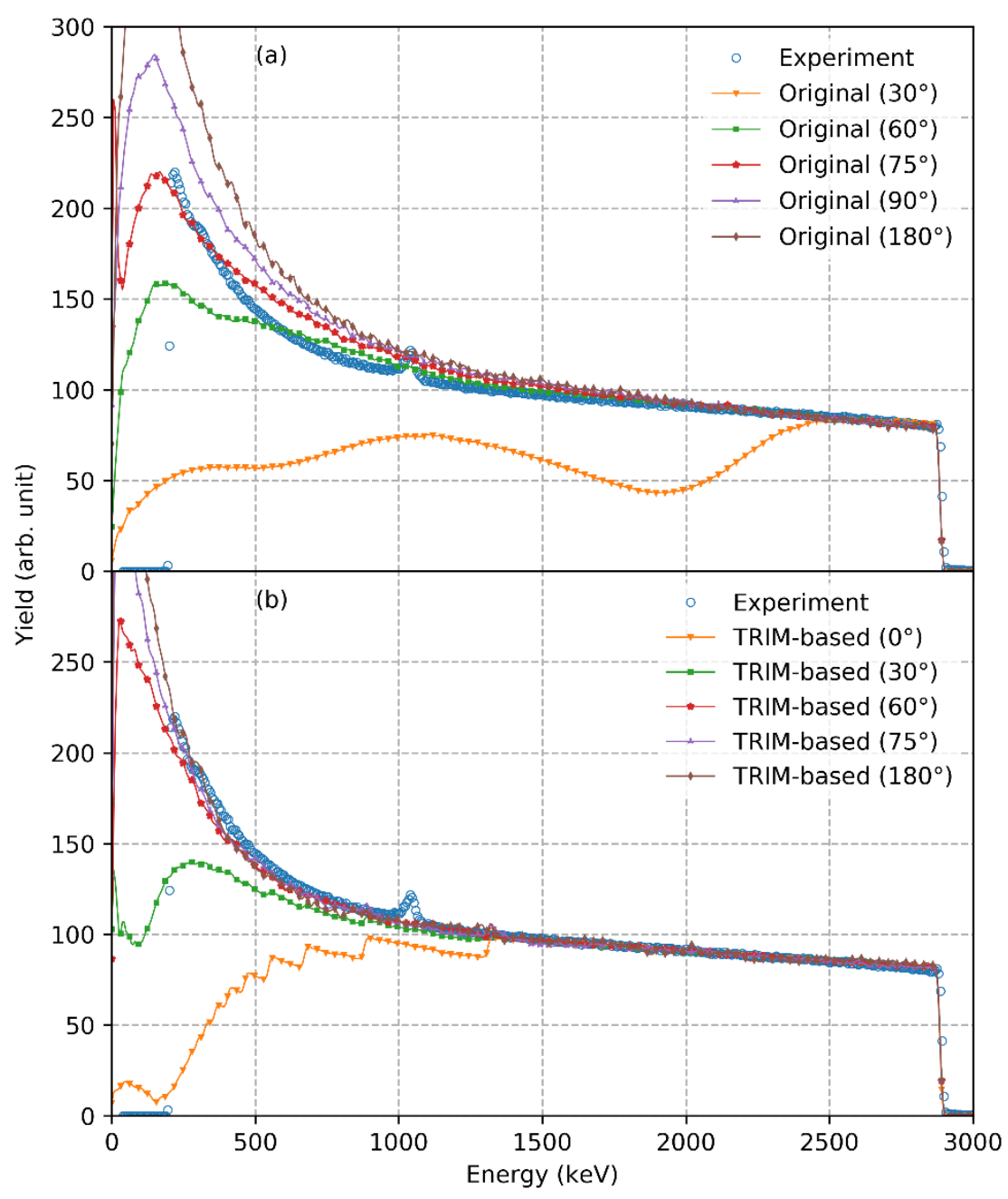

Figure 5. RBS/C spectra of $3.085 \mathrm{MeV} \mathrm{He}^{2+}$ on an $<011>$-oriented UO2 crystal in random directions. Open circles represent experimental spectra. Solid lines with markers correspond to RBSADEC simulated spectra with different spread angles using the original amorphous slowdown approach (top) and the TRIM-based amorphous slowdown approach (bottom). (The peak at around $1.05 \mathrm{MeV}$ in the experimental spectrum is due to the ${ }^{16} \mathrm{O}\left({ }^{4} \mathrm{He},{ }^{4} \mathrm{He}\right){ }^{16} \mathrm{O}$ elastic scattering resonance occuring at $3.038 \mathrm{MeV}[27,36]$, which is not reproduced in RBSADEC simulations.) 


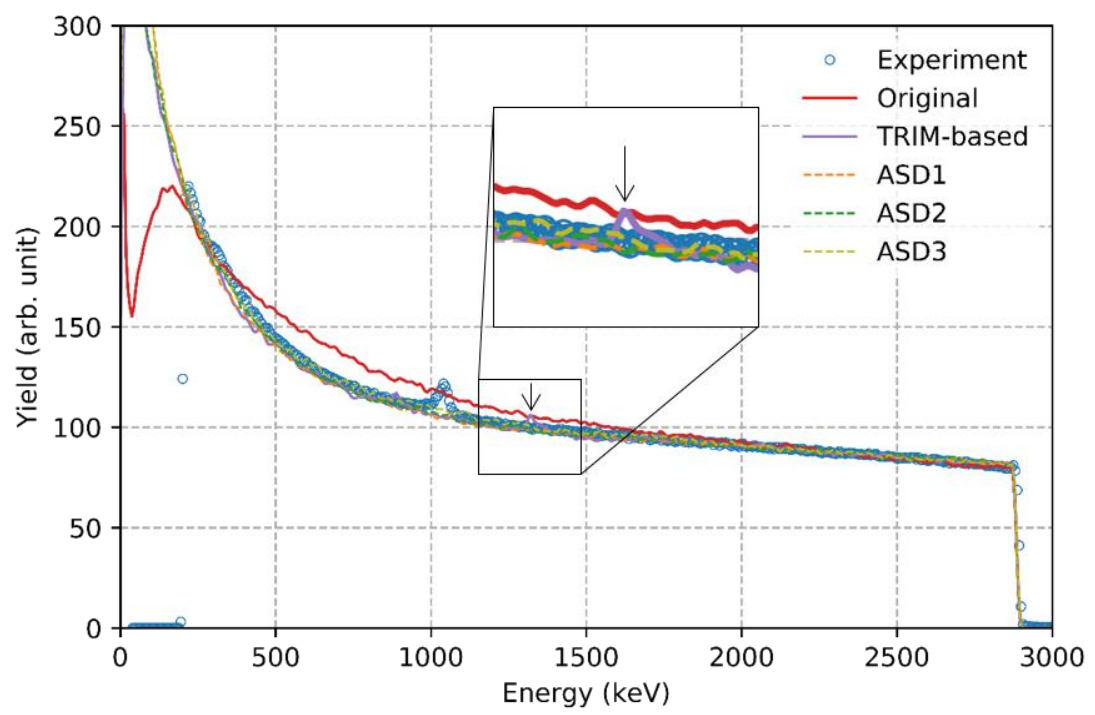

Figure 6. RBS/C random spectra of $3.085 \mathrm{MeV} \mathrm{He}^{2+}$ on an $<011>$-oriented $\mathrm{UO} 2$ crystal obtained from the experiment (open circles) and from the RBSADEC simulations (solid lines) implemented with the original, TRIM-based and ASD(1-3) amorphous slowdown approaches. (The spread angle is set to $75^{\circ}$.) The inset shows the unphysical peak in the simulated spectrum from the TRIM-based approach.

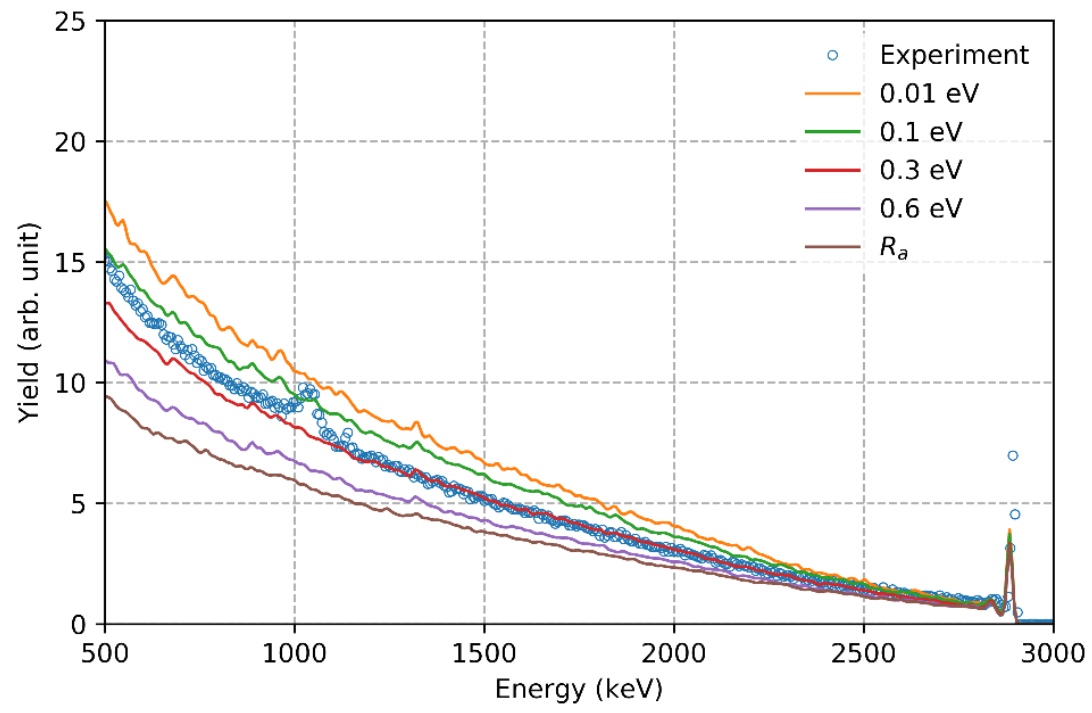

Figure 7. RBS/C aligned spectra of $3.085 \mathrm{MeV} \mathrm{He}^{2+}$ on an $<011>$-oriented UO2 pristine crystal obtained from the experiment (open circles) and from the RBSADEC simulations (solid lines) using the original approach (labeled with $R_{a}$ ) and using the modified approach with different $E_{\min }^{p}$ values. 


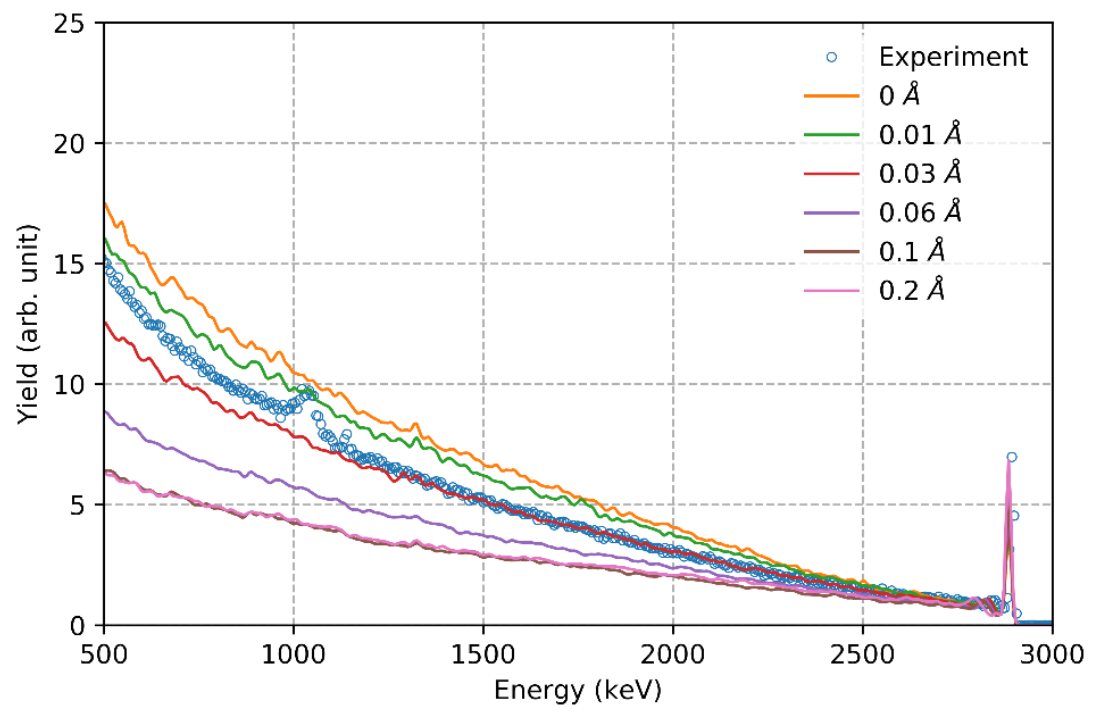

Figure 8. RBS/C aligned spectra of $3.085 \mathrm{MeV} \mathrm{He}^{2+}$ on an $<011>$-oriented UO2 pristine crystal obtained from the experiment (open circles) and from the RBSADEC simulations (solid lines) with different projection interval values. The $E_{\min }^{p}$ value in the simulated spectra is $0.01 \mathrm{eV}$.

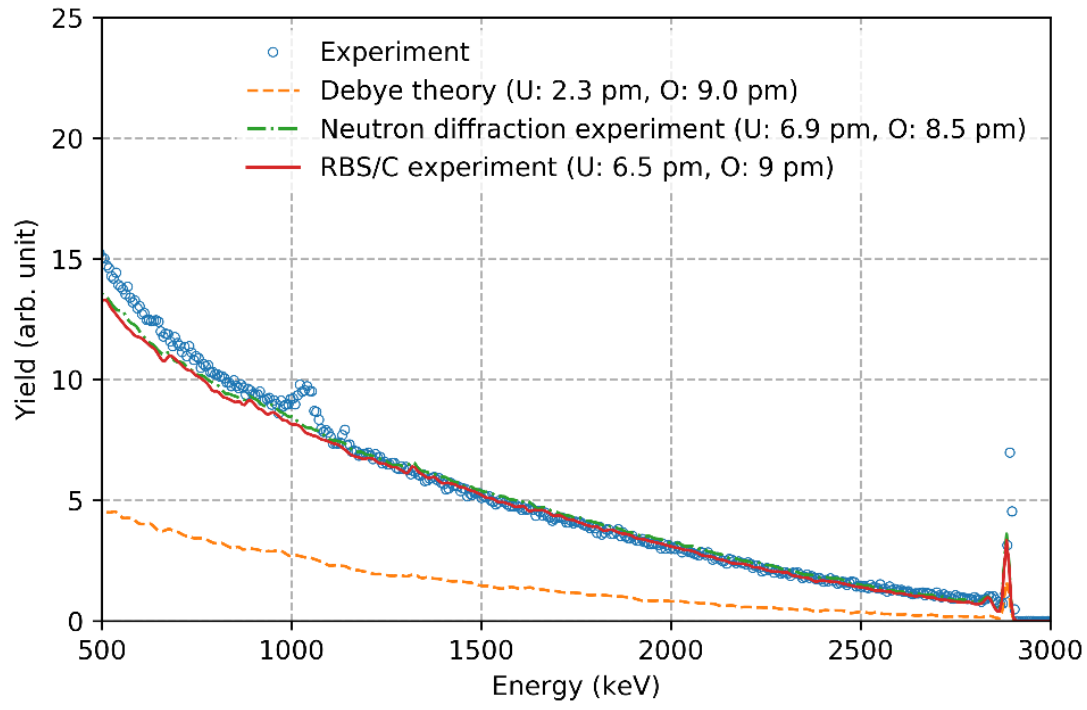

Figure 9. RBS/C aligned spectra of $3.085 \mathrm{MeV} \mathrm{He}^{2+}$ on an $<011>$-oriented UO2 pristine crystal obtained from the experiment (open circles) and from the RBSADEC simulations (lines) with the atom thermal vibration magnitudes obtained from different approaches. 


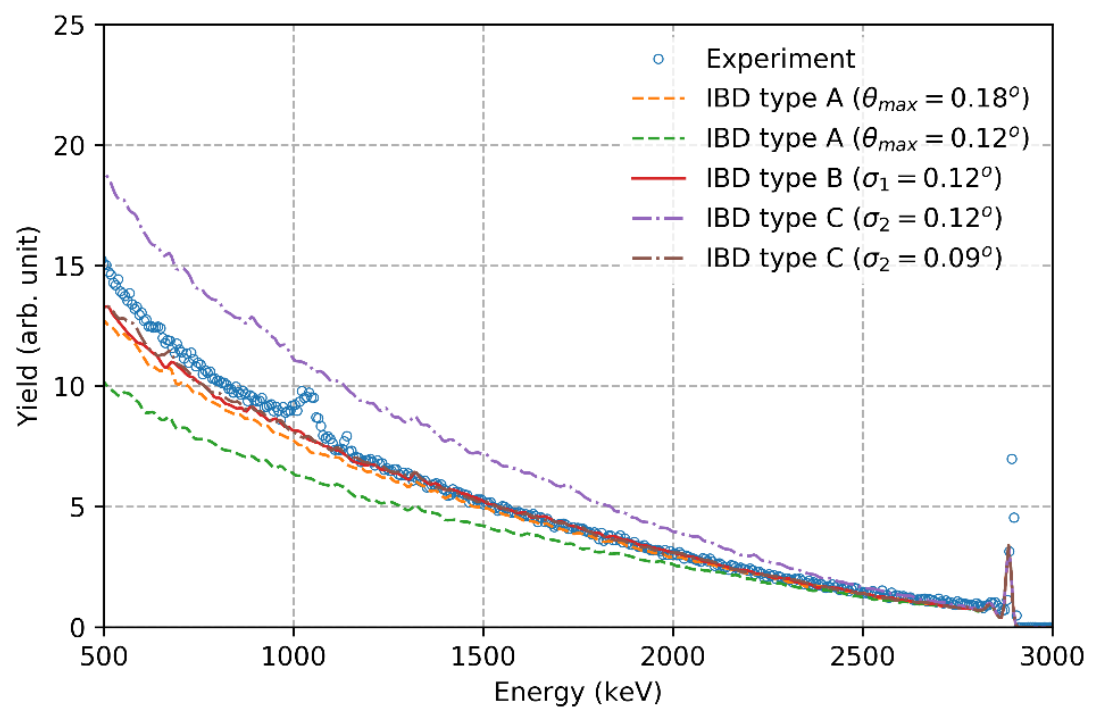

Figure 10. RBS/C aligned spectra of $3.085 \mathrm{MeV} \mathrm{He}^{2+}$ on an $<011>$-oriented UO2 pristine crystal obtained from the experiment (open circles) and from the RBSADEC simulations (lines) with different ion beam divergence types. 


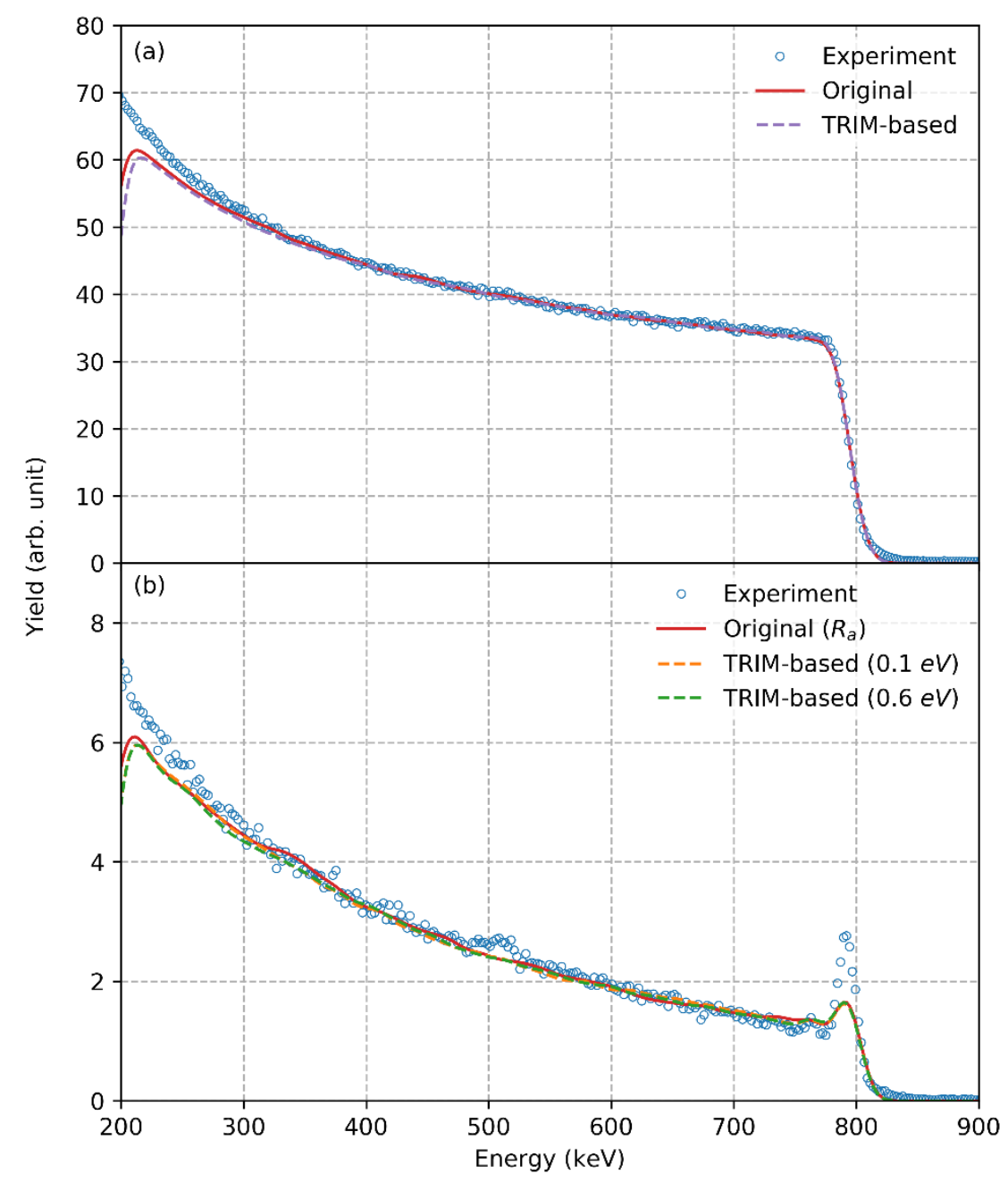

Figure 11. RBS/C spectra of $1.4 \mathrm{MeV} \mathrm{He}{ }^{+}$on an $<001>$-oriented Si crystal. (a) RBS/C random spectra obtained from the experiment and from the RBSADEC simulations implemented with the original and with the TRIM-based amorphous slowdown approaches; (b) RBS/C aligned spectra from the experiment and from the RBSADEC simulations using the original collision partner search method (labeled with $R_{a}$ ) and using the modified collision partner search method (labeled with different $E_{\min }^{p}$ values). In these two figures, open circles represent experimental data, while solid and dashed lines correspond to simulations using the original and the TRIM-based amorphous slowdown approaches, respectively. 


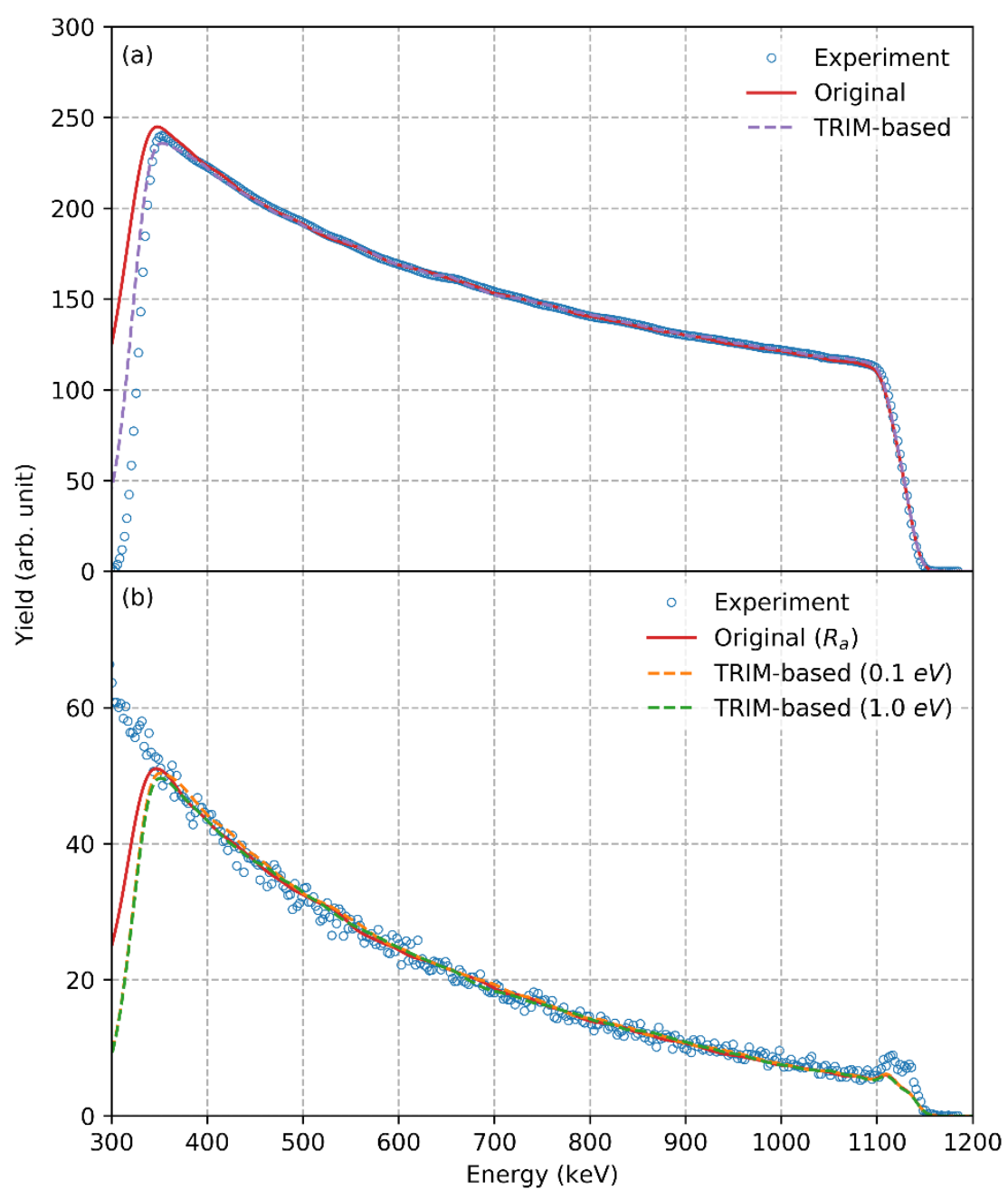

Figure 12. RBS/C spectra of $1.4 \mathrm{MeV} \mathrm{He}^{+}$on an $<001>$-oriented GaAs crystal. (a) RBS/C random spectra obtained from the experiment and from the RBSADEC simulations implemented with the original and with the TRIM-based amorphous slowdown approaches; (b) RBS/C aligned spectra from the experiment and from the RBSADEC simulations using the original collision partner search method (labeled with $R_{a}$ ) and using the modified collision partner search method (labeled with different $E_{\min }^{p}$ values). In these two figures, open circles represent experimental data, while solid and dashed lines correspond to simulations using the original and the TRIM-based amorphous slowdown approaches, respectively. 


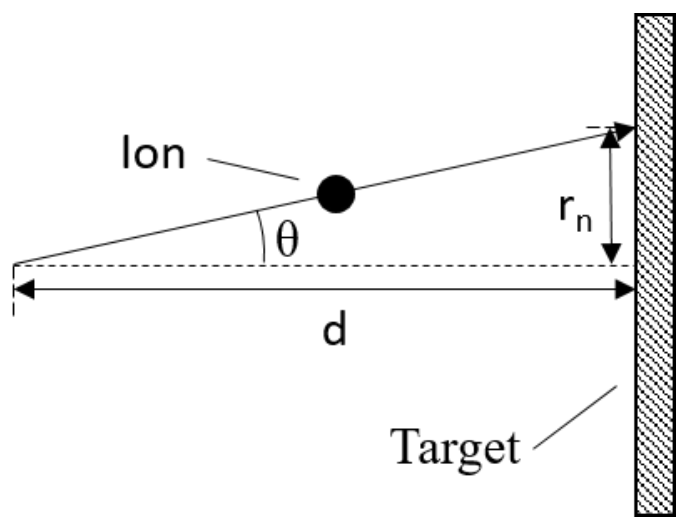

Figure 13. Illustration of the movement of incident ion from the initial position to the target surface

\section{Tables}

Table 1. Characteristics of amorphous slowdown approaches

\begin{tabular}{|c|c|c|c|c|c|}
\hline $\begin{array}{l}\text { Amorphous } \\
\text { slowdown } \\
\text { approach }\end{array}$ & Original & TRIM-based & ASD1 & ASD2 & ASD3 \\
\hline Free flight path & $\begin{array}{c}\text { Random } \\
\text { sampling } \\
\text { (average: } 10 r_{v} \text { ) }\end{array}$ & $\begin{array}{c}\text { Energy } \\
\text { dependent }\left(5^{\circ}\right. \\
\text { mean angular } \\
\text { deflection) }\end{array}$ & $\begin{array}{c}\text { Random } \\
\text { sampling } \\
\text { (average: } 10 r_{v} \text { ) }\end{array}$ & $\begin{array}{l}\text { Fixed } \\
(100 \AA)\end{array}$ & $\begin{array}{l}\text { Fixed } \\
(100 \AA)\end{array}$ \\
\hline $\begin{array}{c}\text { Impact } \\
\text { parameter }\end{array}$ & $\begin{array}{l}\text { Original fixed- } \\
\text { volume }\end{array}$ & $\begin{array}{c}\text { TRIM- } \\
\text { exponential }\end{array}$ & $\begin{array}{c}\text { TRIM- } \\
\text { exponential }\end{array}$ & $\begin{array}{c}\text { TRIM- } \\
\text { exponential }\end{array}$ & $\begin{array}{l}\text { Modified } \\
\text { fixed- } \\
\text { volume }\end{array}$ \\
\hline
\end{tabular}

Table 2. The $\mathrm{U}$ and $\mathrm{O}$ maximum impact parameters with different minimum projectile/target atom interatomic potential energies

\begin{tabular}{ccc}
\hline $\begin{array}{c}\text { Minimum interatomic potential } \\
\text { energy }(\mathrm{eV})\end{array}$ & $\begin{array}{c}\text { Maximum impact parameter for } \\
\text { the U atom }(\AA)\end{array}$ & $\begin{array}{c}\text { Maximum impact parameter for } \\
\text { the O atom }(\AA)\end{array}$ \\
\hline 0.6 & 2.385 & 1.973 \\
0.3 & 2.684 & 2.288 \\
0.1 & 3.191 & 2.846 \\
0.01 & 4.330 & 4.245 \\
\hline \hline
\end{tabular}




\section{References}

[1] M. Nastasi, J.R. Tesmer, Handbook of Modern Ion Beam Materials Analysis, Materials Research Society, Pittsburgh, Pennsylvania,USA, 1995.

[2] G.S. Was, Challenges to the use of ion irradiation for emulating reactor irradiation, J. Mater. Res. 30 (2015) 1158-1182. doi:10.1557/jmr.2015.73.

[3] J.S. Williams, Ion Implantation of Semiconductors, Mater. Sci. Eng. 253 (1998) 8-15. doi:10.1088/0031-9112/27/12/035.

[4] J.H. Barrett, Monte carlo channeling calculations, Phys. Rev. B. 3 (1971) 1527-1547. doi:10.1103/PhysRevB.3.1527.

[5] P.J.M. Smulders, D.O. Boerma, Computer simulation of channeling in single crystals, Nucl. Inst. Methods Phys. Res. B. 29 (1987) 471-489. doi:10.1016/0168-583X(87)90058-9.

[6] J.H. Barrett, Methods of channeling simulation, Nucl. Inst. Methods Phys. Res. B. 44 (1990) 367372. doi:10.1016/0168-583X(90)90652-B.

[7] A. Kling, CASSIS - a new Monte-Carlo computer program for channeling simulation of RBS, NRA and PIXE, Nucl. Instruments Methods Phys. Res. B. 102 (1995) 141-144. doi:https://doi.org/10.1016/0168-583X(95)80131-5.

[8] M. Bianconi, E. Albertazzi, A. Carnera, G. Lulli, R. Nipoti, A. Sambo, RBS-channeling analysis of virgin $6 \mathrm{H}-\mathrm{SiC}$ : Experiments and Monte Carlo simulations, Nucl. Instruments Methods Phys. Res. B. 136-138 (1998) 1267-1271.

[9] L. Nowicki, A. Turos, R. Ratajczak, A. Stonert, F. Garrido, Modern analysis of ion channeling data by Monte Carlo simulations, Nucl. Instruments Methods Phys. Res. Sect. B Beam Interact. with Mater. Atoms. 240 (2005) 277-282. doi:10.1016/j.nimb.2005.06.129.

[10] S. Zhang, K. Nordlund, F. Djurabekova, Y. Zhang, G. Velisa, T.S. Wang, Simulation of Rutherford backscattering spectrometry from arbitrary atom structures, Phys. Rev. E. 94 (2016) 1-12. doi:10.1103/PhysRevE.94.043319.

[11] D.S. Gemmell, Channeling and related effects in the motion of charged particles through crystals, Rev. Mod. Phys. 46 (1974) 129-227. doi:10.1103/RevModPhys.46.129.

[12] S. Zhang, O.H. Pakarinen, M. Backholm, F. Djurabekova, K. Nordlund, J. Keinonen, T.S. Wang, Absence of single critical dose for the amorphization of quartz under ion irradiation, J. Phys. Condens. Matter. 30 (2018) aa9868. doi:10.1088/1361-648X/aa9868.

[13] S. Zhang, K. Nordlund, F. Djurabekova, F. Granberg, Y. Zhang, T.S. Wang, Radiation damage buildup by athermal defect reactions in nickel and concentrated nickel alloys, Mater. Res. Lett. 5 (2017) 433-439. doi:10.1080/21663831.2017.1311284.

[14] C.O. Bacri, C. Bachelet, C. Baumier, J. Bourçois, L. Delbecq, D. Ledu, N. Pauwels, S. Picard, S. Renouf, C. Tanguy, SCALP, a platform dedicated to material modifications and characterization under ion beam, Nucl. Instruments Methods Phys. Res. Sect. B Beam Interact. with Mater. Atoms. 406 (2017) 48-52. doi:10.1016/j.nimb.2017.03.036. 
[15] J.F. Ziegler, J.P. Biersack, M.D. Ziegler, The Stopping and Range of Ions in Matter, Chester, Maryland, 2015.

[16] K. Arstila, T. Sajavaara, J. Keinonen, Monte Carlo simulation of multiple and plural scattering in elastic recoil detection, Nucl. Instruments Methods Phys. Res. Sect. B Beam Interact. with Mater. Atoms. 174 (2001) 163-172. doi:10.1016/S0168-583X(00)00435-3.

[17] J.P. Biersack, L.G. Haggmark, A Monte Carlo computer program for the transport of energetic ions in amorphous targets, Nucl. Instruments Methods. 174 (1980) 257-269. doi:10.1016/0029554X(80)90440-1.

[18] C. Borschel, C. Ronning, Ion beam irradiation of nanostructures - A 3D Monte Carlo simulation code, Nucl. Instruments Methods Phys. Res. Sect. B Beam Interact. with Mater. Atoms. 269 (2011) 2133-2138. doi:10.1016/j.nimb.2011.07.004.

[19] J. Crocombette, C. Van Wambeke, Quick calculation of damage for ion irradiation : implementation in Iradina and comparisons to SRIM, 7 (2019).

[20] B. Yuan, P.C. Yu, S.M. Tang, A database method for binary atomic scattering angle calculation, Nucl. Inst. Methods Phys. Res. B. 83 (1993) 413-418. doi:10.1016/0168-583X(93)95864-2.

[21] K.E. Atkinson, An Introduction to numerical analysis, 2nd ed., John Wiley \& Sons, New York, 1989.

[22] M. Robinson, I. Torrens, Computer simulation of atomic-displacement cascades in solids in the binary-collision approximation, Phys. Rev. B. 9 (1974) 5008-5024. doi:10.1103/PhysRevB.9.5008.

[23] M. Posselt, Crystal-TRIM and its application to investigations on channeling effects during ion implantation, Radiat. Eff. Defects Solids. 130-131 (1994) 87-119.

[24] E. Albertazzi, G. Lulli, Monte Carlo simulation of ion implantation in crystalline SiC, Nucl. Instruments Methods Phys. Res. Sect. B Beam Interact. with Mater. Atoms. 120 (1996) 147-150.

[25] K. Nordlund, G. Hobler, Dependence of ion channeling on relative atomic number in compounds, Nucl. Instruments Methods Phys. Res. Sect. B Beam Interact. with Mater. Atoms. 435 (2018) 6169. doi:10.1016/j.nimb.2017.11.020.

[26] B.T.M. Willis, Neutron diffraction studies of the actinide oxides II. Thermal motions of the atoms in uranium dioxide and thorium dioxide between room temperature and $1100{ }^{\circ} \mathrm{C}$, Proc. R. Soc. London. Ser. A. Math. Phys. Sci. 274 (2006) 134-144. doi:10.1098/rspa.1963.0118.

[27] F. Garrido, L. Nowicki, L. Thomé, Channeling investigation of the crystalline structure of U4 O9y, Phys. Rev. B - Condens. Matter Mater. Phys. 74 (2006) 1-13. doi:10.1103/PhysRevB.74.184114.

[28] J.O. Kephart, B.L. Berman, R.H. Pantell, S. Datz, R.K. Klein, H. Park, Thermal-vibrational amplitudes of silicon determined by channeling-radiation measurements, Phys. Rev. B. 44 (1991) 1992-2002. doi:https://doi.org/10.1103/PhysRevB.44.1992.

[29] M.T. Robinson, Slowing-down time of energetic atoms in solids, Phys. Rev. B. 40 (1989).

[30] G. Dolling, R.A. Cowley, A.D.B. Woods, The Crystal Dynamics of Uranium Dioxide, Can. J. Phys. 43 (1965) 1397-1413. 
[31] B.T.M. Willis, R.G. Hazell, Re-analysis of single-crystal neutron-diffraction data on UO2 using third cumulants, Acta Crystallogr. Sect. A. 36 (1980) 582-584. doi:10.1107/S0567739480001246.

[32] A. Turos, P. Jozwik, L. Nowicki, N. Sathish, Ion channeling study of defects in compound crystals using Monte Carlo simulations, Nucl. Instruments Methods Phys. Res. Sect. B Beam Interact. with Mater. Atoms. 332 (2014) 50-55. doi:10.1016/j.nimb.2014.02.028.

[33] A.W. Stevenson, Thermal vibrations and bonding in GaAs: an extended-face crystal study, Acta Crystallogr. Sect. A. 50 (1994) 621-632. doi:10.1107/S0108767393013947.

[34] N.M. Butt, J. Bashir, M.N. Khan, Compilation of temperature factors of cubic compounds, Acta Crystallogr. Sect. A. 49 (1993) 171-174. doi:10.1107/S0108767392006974.

[35] O.F. Toader, F.U. Naab, Beam Profiling and Measurement At MIBL, Proc. DIPAC09, Basel, Switz. (2009) 333-335.

[36] R.J. Cameron, Elastic Scattering of Alpha-Particles by Oxygen, Phys. Rev. 90 (1953). doi:https://doi.org/10.1103/PhysRev.90.839. 